\title{
Special issue on sensing and mobility in pervasive computing
}

\author{
Francesco Marcelloni • Daniele Puccinelli • \\ Alessio Vecchio
}

Published online: 19 February 2013

(C) Springer-Verlag Berlin Heidelberg 2013

The goal of pervasive systems is to create technologies and applications that are transparent to the user. As a consequence, properly implemented pervasive systems need to acquire most of their needed information independently, contacting the user only in exceptional circumstances. This objective can be achieved by exploiting the sensing capabilities of mobile smart devices and/or by leveraging dedicated sensing devices. In recent years, the specific challenges introduced by mobility and sensing in pervasive computing have been the focus of a rich body of work.

This special issue includes articles on sensing and mobility that represent extended versions of papers originally presented in two IEEE PerCom 2012 workshops, namely the 8th International Workshop on sensor networks and systems for pervasive computing (PerSeNS 2012) and the First International Workshop on the impact of human mobility in pervasive systems and applications (PerMoby 2012). PerSeNS focuses on wireless sensor networks as the basic building block of pervasive computing environments. PerMoby explores the impact of human mobility on pervasive applications, systems and protocols.

F. Marcelloni $(\bowtie) \cdot$ A. Vecchio

University of Pisa, Pisa, Italy

e-mail: francesco.marcelloni@iet.unipi.it

A. Vecchio

e-mail: alessio.vecchio@iet.unipi.it

D. Puccinelli

University of Applied Sciences of Southern Switzerland,

Switzerland, Switzerland

e-mail: daniele.puccinelli@supsi.ch
Seven selected papers were submitted to the special issue. Every paper was reviewed by three referees and, after two rounds of review, finally five of them were accepted according to the referees' evaluations.

The first paper, "Discovering Events in the City Via Mobile Network Analysis", presents a methodology to discover events from human mobility patterns captured by mobile network usage. The results, obtained with an extensive dataset provided by a telecom operator, show that the proposed approach is effective and versatile.

The second paper, "Location Prediction and Mobility Modeling for Enhanced Localization Solution”, presents a solution for continuous user localization that combines standard location tracking techniques, newly built-in technologies, human mobility modeling and machine learning methods in order to reduce energy consumption and preserve user privacy.

The third paper, "An Analysis of Distance Estimation to Detect Proximity in Social Interactions", presents a mobile platform for user proximity detection that estimates the distance between users by applying data mining techniques to the analysis of the Wi-Fi received signal strength.

The fourth paper, "Route Selection for Mobile Sensor Nodes on Public Transport Networks", describes a strategy for maximizing the sensing coverage of a mobile sensor network consisting of nodes mounted on public transport vehicles, while providing a sufficient number of checkpoints, that is, points where vehicles are in the vicinity of each other for comparing sensor readings and thus increasing robustness.

The last paper, "Leveraging the Robustness of Genetic Networks: A Case Study on Bio-inspired Wireless Sensor Network Topologies", proposes a biologically-inspired approach based on Gene Regulatory Networks (GRNs) of 
the organism E. coli to address packet loss in wireless sensor networks.

Finally, as Guest Editors of this special issue, we would like to thank all the authors for their contributions and the referees for their outstanding cooperation and constructive input.
The Guest Editors:

Francesco Marcelloni, University of Pisa, Italy.

Daniele Puccinelli, University of Applied Sciences of Southern Switzerland, Switzerland.

Alessio Vecchio, University of Pisa, Italy. 